\title{
Optimal Factorization of the State-Dependent Riccati Equation Technique in a Satellite Attitude and Orbit Control System
}

\author{
ALESSANDRO GERLINGER ROMERO \\ Space Mechanics and Contro Division \\ Brazilian National Institute for Spaece Research \\ Astronautas Avenue, 1758 - São José dos Campos \\ BRAZIL \\ LUIZ CARLOS GADELHA DE SOUZA \\ Engineering Center \\ Federal University of ABC \\ dos Estados Avenue - São Bernardo do Campo \\ BRAZIL
}

\begin{abstract}
The satellite attitude and orbit control system (AOCS) can be designed with success by linear control theory if the satellite has slow angular motions and small attitude maneuver. However, for large and fast maneuvers, the linearized models are not able to represent all the perturbations due to the effects of the nonlinear terms present in the dynamics and in the actuators (e.g., saturation). Therefore, in such cases, it is expected that nonlinear control techniques yield better performance than the linear control techniques. One candidate technique for the design of AOCS control law under a large maneuver is the State-Dependent Riccati Equation (SDRE). SDRE entails factorization (that is, parameterization) of the nonlinear dynamics into the state vector and the product of a matrix-valued function that depends on the state itself. In doing so, SDRE brings the nonlinear system to a (nonunique) linear structure having state-dependent coefficient (SDC) matrices and then it minimizes a nonlinear performance index having a quadratic-like structure. The nonuniqueness of the SDC matrices creates extra degrees of freedom, which can be used to enhance controller performance, however, it poses challenges since not all SDC matrices fulfill the SDRE requirements. Moreover, regarding the satellite's kinematics, there is a plethora of options, e.g., Euler angles, Gibbs vector, modified Rodrigues parameters (MRPs), quaternions, etc. Once again, some kinematics formulation of the AOCS do not fulfill the SDRE requirements. In this paper, we evaluate the factorization options (SDC matrices) for the AOCS exploring the requirements of the SDRE technique. Considering a Brazilian National Institute for Space Research (INPE) typical mission, in which the AOCS must stabilize a satellite in three-axis, the application of the SDRE technique equipped with the optimal SDC matrices can yield gains in the missions. The initial results show that MRPs for kinematics provides an optimal SD
\end{abstract}

Key-Words: SDRE, nonlinear, control, attitude

Received: December 6, 2020. Revised: April 4, 2021. Accepted: April 16, 2021. Published: April 26, 2021.

\section{Introduction}

The design of a satellite attitude and orbit control subsystem (AOCS) that involves plant uncertainties, large angle maneuvers and fast attitude control following a stringent pointing, requires nonlinear control methods to satisfy performance and robustness requirements.

An example is a typical mission of the Brazilian National Institute for Space Research (INPE), in which the AOCS must stabilize a satellite in threeaxes so that the optical payload can point to the desired target with few arcsecs of pointing accuracy.

One candidate method for a nonlinear AOCS control law is the State-Dependent Riccati Equation
(SDRE) method, originally proposed by [1] and then explored in detail by [2, 3, 4]. SDRE provides an effective algorithm for synthesizing nonlinear feedback control by allowing nonlinearities in the system states while offering great design flexibility through state-dependent weighting matrices. SDRE entails factorization (that is, parameterization) of the nonlinear dynamics into the state vector and the product of a matrix-valued function that depends on the state itself. In doing so, SDRE brings the nonlinear system to a (nonunique) linear structure having state-dependent coefficient (SDC) matrices and then it minimizes a nonlinear performance index having a quadratic-like structure. 
Accordingly, a suboptimal control law is carried out by a real-time solution of an algebraic Riccati equation (ARE) using the SDC matrices by means of a numerical algorithm. Therefore, SDRE linearizes the plant about the instantaneous point of operation and produces a constant state-space model of the system. The process is repeated in the next sampling steps, producing and controlling several state dependent linear models out of a nonlinear one.

In this paper, we evaluate the factorization options (SDC matrices) for the AOCS exploring the requirements of the SDRE technique. In particular, the nonuniqueness of the SDC matrices creates extra degrees of freedom, which can be used to enhance controller performance, however, it poses challenges since not all SDC matrices fulfill the SDRE requirements. Moreover, regarding the satellite's kinematics, there is a plethora of options, e.g., Euler angles, Gibbs vector, modified Rodrigues parameters (MRPs), quaternions, etc. Once again, some kinematics formulation of the AOCS do not fulfill the SDRE requirements.

A good survey of the SDRE method can be found in [2] and its systematic application to deal with a nonlinear plant in [3]. The SDRE method was applied by [5, 6, 7, 8, 9] for controlling a nonlinear system similar to the six-degree of freedom satellite model considered in this paper. [5] defined a simulator using Euler angles based on commercial software, whereas, [6, 7] applied quaternions on commercial software. [9] extended an opensource project and defined an opensource simulator based on Euler angles.

The application of SDRE method, and, consequently, the ARE problem that arises, have already been studied in the available literature, e.g., [8] investigated the approaches for the ARE solving as well as the resource requirements for such online solving. Recently, [6] proposed the usage of differential algebra to reduce the resource requirements for the realtime implementation of SDRE controllers. In fact, the intensive resource requirements for the online ARE solving is the major drawback of SDRE. Nonetheless, the SDRE method has three major advantages: (a) simplicity, (b) numerical tractability and (c) flexibility for the designer, being comparable to the flexibility in the LQR [7].

To the best of our knowledge, since SDC matrices are nonunique, there is no work focused on the optimal arrangement of the SDC for the satellite attitude control stabilization. Such optimal arrangement has the potential to increase performance and enhance robustness. Therefore, the first contribution of the present paper is the explicit modeling of the state-space model for a three-axes stabilized attitude-maneuvering satellite using quaternions, Gibbs vector and modified Rodrigues param- eters (MRPs). The second, and most important, is the evaluation of which of these models is the optimal factorization of the SDRE technique in an AOCS with nonlinear dynamics for a given Monte Carlo perturbation model based on a set of parameters, initial conditions and references for the controller.

The models are evaluated for an attitude maneuver called the upside-down in the launch and early orbit phase (LEOP). In LEOP, the AOCS must dump the residual angular velocity and point the satellite solar panels towards the Sun. The results shown that MRPs provides better performance in the set of scenarios considered.

This paper is organized as follows. In next section, the problem is described. Afterwards, the satellite is modelled and the basic equations are shared. In the sequel, the state-space models are presented. Finally, the controllability of such models is explored as well as parametrization are evaluated. At the end, conclusions are presented.

\section{Problem Description}

The SDRE technique entails factorization (that is, parametrization) of the nonlinear dynamics into the state vector and the product of a matrix-valued function that depends on the state itself. In doing so, SDRE brings the nonlinear system to a (nonunique) linear structure having SDC matrices given by Equation 11

$$
\begin{array}{r}
\overrightarrow{\dot{x}}=A(\vec{x}) \vec{x}+B(\vec{x}) \vec{u} \\
\vec{y}=C \vec{x}
\end{array}
$$

where $\vec{x}$ is the state vector and $\vec{u}$ is the control vector. The SDC form has the same structure as a linear system, but with the system matrices, $A$ and $B$, being functions of the state vector. The nonuniqueness of the SDC matrices creates extra degrees of freedom, which can be used to enhance controller performance, however, it poses challenges since not all SDC matrices fulfill the SDRE requirements, e.g., the pair $(A, B)$ must be pointwise stabilizable.

The system model in Equation 1 is subject of the cost functional described in Equation 2

$$
J\left(\overrightarrow{x_{0}}, \vec{u}\right)=\frac{1}{2} \int_{0}^{\infty}\left(\vec{x}^{T} Q(\vec{x}) \vec{x}+\vec{u}^{T} R(\vec{x}) \vec{u}\right) d t
$$

where $Q(\vec{x}) \in \mathrm{R}^{n \times n}$ and $R(\vec{x}) \in \mathrm{R}^{m \times m}$ are the state-dependent weighting matrices. In order to ensure local stability, $Q(\vec{x})$ is required to be positive semi-definite for all $\vec{x}$ and $R(\vec{x})$ is required to be positive for all $\vec{x}[\underline{8}]$.

The SDRE controller linearizes the plant about the current operating point and creates constant state space matrices so that the LQR method can be used. 
This process is repeated in all samplings steps, resulting in a pointwise linear model from a non-linear model, so that an ARE is solved and a control law is computed also in each step.

Therefore, according to LQR theory and Equation 1 and Equation 2, the state-feedback control law in each sampling step is $\vec{u}=-K(\vec{x}) \vec{x}$ and the statedependent gain $K(\vec{x})$ is obtained by Eq. 3 [3] .

$$
K(\vec{x})=R^{-1}(\vec{x}) B^{T}(\vec{x}) P(\vec{x})
$$

where $P(\vec{x})$ is the unique, symmetric, positivedefinite solution of the algebraic state-dependent Riccati equation (SDRE) given by Equation 4[ [3].

$$
\begin{array}{r}
P(\vec{x}) A(\vec{x})+A^{T}(\vec{x}) P(\vec{x}) \\
-P(\vec{x}) B(\vec{x}) R^{-1}(\vec{x}) B^{T}(\vec{x}) P(\vec{x}) \\
+Q(\vec{x})=0
\end{array}
$$

Considering that Equation 4 is solved in each sampling step, it is reduced to an ARE.

Finally, among other conditions [3], for the application of the SDRE technique in a given system model, the following one is the cornerstone for the current paper: $\operatorname{pair}(A, B)$ is pointwise stabilizable (a sufficient test for stabilizability is to check the rank of the controllability matrix).

\section{Satellite Physical Modeling}

The satellite model is designed based on the Amazonia-1, a typical mission developed by INPE, in which the AOCS must stabilize the satellite in threeaxis so that the optical payload can point to the desired target. Therefore, the satellite model is defined to be a three-axis stabilized, attitude-maneuvering satellite, a zero-bias-momentum system. A major control requirement is to remove the unwanted accumulated angular momentum, which would drive the satellite pointing away from the desired target. Thus, an active control system is needed to dump the residual body angular velocity that is created by perturbation torques from the space environment and launch vehicles [10, 11].

The satellite model available, which is based on the Amazonia-1, uses reaction wheels (momentum exchange actuators) to provide fine attitude control and to maneuver the satellite [11].

The simulator models two types of sensors to compute and propagate the attitude: (1) a set of Sun sensors, and (2) a gyro, which provide all the necessary information for the LEOP attitude maneuver to acquire the Sun pointing. The sensor models are ideal and simplified. In other words, they can read the physical quantities at any moment with infinite accuracy.

The kinematics and rotational dynamics are briefly shared in the next subsections.

\subsection{Kinematics}

The satellite attitude is represented by means of quaternions in the model. Hence, the dynamic equation of the quaternion $Q$ that rotates the ECI reference frame into alignment with the satellite body reference frame is Equation 5[ [10].

$$
\begin{array}{r}
\dot{Q}=\frac{1}{2} \Omega(\vec{\omega}) Q=\frac{1}{2} \Xi(Q) \vec{\omega} \\
\Omega(\vec{\omega}):=\left[\begin{array}{cccc}
0 & \omega_{3} & -\omega_{2} & \omega_{1} \\
-\omega_{3} & 0 & \omega_{1} & \omega_{2} \\
\omega_{2} & -\omega_{1} & 0 & \omega_{3} \\
-\omega_{1} & -\omega_{2} & -\omega_{3} & 0
\end{array}\right] \\
\Xi(Q):=\left[\begin{array}{ccc}
q_{4} & -q_{3} & q_{2} \\
q_{3} & q_{4} & -q_{1} \\
-q_{2} & q_{1} & q_{4} \\
-q_{1} & -q_{2} & -q_{3}
\end{array}\right],
\end{array}
$$

where $\vec{\omega}=\left[\omega_{1} \omega_{2} \omega_{3}\right]^{T}$ is the angular velocity measured in the satellite body reference frame.

Equation 5 can be rearranged in Equation 6 , using the vector $g$ (Gibbs vector or Rodrigues parameter) as $Q=\left[g^{T} \mid q_{4}\right]^{T}$.

$$
\dot{Q}=-\frac{1}{2}\left[\begin{array}{l}
\omega^{\times} \\
\omega^{T}
\end{array}\right]\left[\begin{array}{l}
q_{1} \\
q_{2} \\
q_{3}
\end{array}\right]+\frac{1}{2} q_{4}\left[\begin{array}{c}
I_{3 \times 3} \\
0
\end{array}\right] \omega
$$

Once more Equation 6 can be written as in Equation 7. using the vector $p=\frac{g}{1+q_{4}}$ (Modified Rodrigues Parameters).

$$
\dot{p}=\frac{1}{2}\left[\frac{1}{2}\left(1-p^{T} p\right) I_{3 \times 3}+p^{\times}+p p^{T} I_{3 \times 3}\right] \omega
$$

\subsection{Rotational Dynamics}

The model of the rotational dynamics of the satellite is based on the Euler-Newton formulation of the rotational motion and in the definition that the satellite has a set of 3 reaction wheels, each one aligned with its principal axes of inertia. One can define the inertia moment of the satellite coupled with the 3 reaction wheels $\left(I_{b}\right)$ using the Equation 8

$$
I_{b}:=I-\sum_{n=1}^{3} I_{n, s} a_{n} a_{n}^{T}
$$

where $I$ is the inertia moment of the satellite, $I_{n, s}$ is the inertia moment of the reaction wheels in their symmetry axis $a_{n}$.

Assuming that there is no net external torque and using Equation 8 , the rotational dynamics of the satellite is modeled by Equation 9

$$
\begin{array}{r}
\dot{\vec{\omega}}^{b}=I_{b}^{-1} g_{c m}^{\vec{c}}-I_{b}^{-1} \omega^{\times} I_{b} \vec{\omega} \\
-I_{b}^{-1} \omega^{\times} \sum_{n=1}^{3} h_{w, n} \overrightarrow{a_{n}}-I_{b}^{-1} \sum_{n=1}^{3} g_{n} \overrightarrow{a_{n}}
\end{array}
$$


where, $\omega$ is the angular velocity, $g_{n}$ is the torque generated by the $n$ reaction wheel and $h_{w, n}$ is the angular momentum of the $n$ reaction wheel about its center of mass.

\section{Nonlinear Control Based on State-Dependent Riccati Equation (SDRE)}

In such zero-bias-momentum system, there are two dynamics states that must be controlled: (1) the attitude (perhaps described by unit quaternions $Q$ ) and (2) its stability (derivative of $Q$, in other words, the angular velocity, $\omega$, of the satellite). Considering the satellite model, the following high-level requirements are derived: (1) is refined in "the attitude must be stabilized and must follow the sun according to a given sun vector in the satellite" and (2) is refined in "the angular velocity read by the gyroscope must be as close as possible of $0 "$. These are typical requirements of the LEOP, in most of missions the most critical phase, in which the demand for the reaction wheels is the highest one.

The state and the control vectors, for the control loop, can be defined by Equation 10 .

$$
\begin{array}{r}
{\left[\begin{array}{l}
\overrightarrow{x_{1}} \\
\overrightarrow{x_{2}}
\end{array}\right]=\left[\begin{array}{l}
Q \\
\vec{\omega}
\end{array}\right]} \\
{\left[\overrightarrow{u_{1}}\right]=\left[\sum_{n=1}^{3} g_{n} \overrightarrow{a_{n}}\right]}
\end{array}
$$

The following subsections presents the state-space models.

\subsection{Quaternions}

Taking into account the control vector defined in Equation 10, the state space model can be defined using Equation 5 and Equation 9 by the equations 11 and 12

$$
\begin{aligned}
& {\left[\begin{array}{c}
\dot{x_{1}} \\
\dot{x_{2}}
\end{array}\right]=\left[\begin{array}{cc}
\frac{1}{2} \Omega & 0 \\
0 & -I_{b}^{-1} \omega^{\times} I_{b}+I_{b}^{-1}\left(\sum_{n=1}^{3} h_{w, n} a_{n}\right)^{\times}
\end{array}\right]\left[\begin{array}{l}
x_{1} \\
x_{2}
\end{array}\right]+\left[\begin{array}{c}
0 \\
-I_{b}^{-1}
\end{array}\right]\left[u_{1}\right]} \\
& {[y]=I\left[\begin{array}{l}
x_{1} \\
x_{2}
\end{array}\right]} \\
& {\left[\begin{array}{c}
\dot{x_{1}} \\
\dot{x_{2}}
\end{array}\right]=\left[\begin{array}{cc}
0 & \frac{1}{2} \Xi \\
0 & -I_{b}^{-1} \omega^{\times} I_{b}+I_{b}^{-1}\left(\sum_{n=1}^{3} h_{w, n} a_{n}\right)^{\times}
\end{array}\right]\left[\begin{array}{l}
x_{1} \\
x_{2}
\end{array}\right]+\left[\begin{array}{c}
0 \\
-I_{b}^{-1}
\end{array}\right]\left[u_{1}\right]} \\
& {[y]=I\left[\begin{array}{l}
x_{1} \\
x_{2}
\end{array}\right]}
\end{aligned}
$$

Nonetheless, such state-space models, in Equation 11 and Equation 12, do not fulfill the SDRE requirements, in particular, the $\operatorname{pair}(A, B)$ is not pointwise stabilizable. Therefore, it is impossible to use such models with SDRE technique.

\subsection{Gibbs Vector}

An option for the definition of the state-space model is to use Equation 6, which leads to Equation 13.

$$
\begin{aligned}
& {\left[\begin{array}{c}
\dot{x_{1}} \\
\dot{x_{2}}
\end{array}\right]=\left[\begin{array}{ccc}
-\frac{1}{2}\left[\begin{array}{c}
\omega^{\times} \\
\omega^{T}
\end{array}\right] & 0 & {\left[\begin{array}{c}
\frac{1}{2} q_{4} I_{3 \times 3} \\
0
\end{array}\right]} \\
0 & 0 & -I_{b}^{-1} \omega^{\times} I_{b}+I_{b}^{-1}\left(\sum_{n=1}^{3} h_{w, n} a_{n}\right)^{\times}
\end{array}\right]\left[\begin{array}{l}
x_{1} \\
x_{2}
\end{array}\right]+\left[\begin{array}{c}
0 \\
-I_{b}^{-1}
\end{array}\right]\left[u_{1}\right]} \\
& {[y]=I\left[\begin{array}{l}
x_{1} \\
x_{2}
\end{array}\right]}
\end{aligned}
$$


Equation 13 has been shown to satisfy SDRE conditions, moreover, in it only $A$ is a function of the state vector, consequently, $A(\vec{x})$.

\subsection{Modified Rodrigues Parameters (MRPs)}

Another alternative for the definition of the statespace model is to use Equation 7 , which leads to Equation 14

$$
\begin{array}{r}
{\left[\begin{array}{c}
x_{3} \\
x_{2}
\end{array}\right]=\left[\begin{array}{c}
p \\
\vec{\omega}
\end{array}\right]} \\
{\left[\begin{array}{c}
\dot{x_{3}} \\
\dot{x_{2}}
\end{array}\right]=\left[\begin{array}{cc}
-\frac{1}{2} \omega^{\times}+\frac{1}{2}\left(\omega^{T} p\right) I_{3 \times 3} & \frac{1}{4}\left(1-p^{T} p\right) I_{3 \times 3} \\
0 & -I_{b}^{-1} \omega^{\times} I_{b}+I_{b}^{-1}\left(\sum_{n=1}^{3} h_{w, n} a_{n}\right)^{\times}
\end{array}\right]\left[\begin{array}{c}
x_{3} \\
x_{2}
\end{array}\right]+\left[\begin{array}{c}
0 \\
-I_{b}^{-1}
\end{array}\right]\left[\begin{array}{c}
u_{1} \\
\end{array}\right]} \\
{[y]=I\left[\begin{array}{l}
x_{3} \\
x_{2}
\end{array}\right]}
\end{array}
$$

Equation 14 has been shown to satisfy SDRE conditions.

\section{Controllability Comparison}

According to [3], an effective approach for selecting the optimal state-space model for the SDRE is to attempt to maximize the pointwise stabilizability of the possible models, since pointwise control effort can be directly linked with controllability. Controllability criterion requires the value of determinant of the controllability matrix to be different from zero, therefore, a graphical comparison of the absolute value of the determinant of controllability matrix can be used to reveal when pointwise controllability is maximized. For multi-input systems, as the one studied in the present paper, the controllability matrix is nonsquare, then the controllability matrix multiplied by its transpose is used to evaluate the determinant.

In order to compare the performance of the controllers, a simulation test was conducted with the full Monte Carlo perturbation model described as follows: (1) the initial Euler angle errors of the nonlinear spacecraft system are randomly selected using independent normal distributions $(\mu=0, \sigma=1)$ multiplied by $90^{\circ}$; and (2) the initial angular velocity errors are randomly selected using independent normal distributions $(\mu=0, \sigma=1)$ multiplied by $0.11 \mathrm{rad} / \mathrm{s}$.

Numerical simulations were performed to determine which of the equations (13) or 14) maximizes the controllability of the system for the given satellite characteristics, initial conditions and references in Table 1 . In order to compare the controllability, a full Monte Carlo perturbation model was conducted with two independent systems each one defined by equations 13 and 14 applying a SDRE controller $(Q=1$ and $R=1$ ). The Monte Carlo perturbation model is described as follows: (1) the initial Euler angle errors of the nonlinear spacecraft system are randomly

\begin{tabular}{|c|c|}
\hline Name & Value \\
\hline \multicolumn{2}{|l|}{ Satellite Characteristics } \\
\hline inertia tensor $\left(\mathrm{kg} \cdot \mathrm{m}^{2}\right)$ & $\begin{array}{c}\operatorname{diag}(310.0 \\
360.0,530.7)\end{array}$ \\
\hline \multicolumn{2}{|c|}{ Actuators Characteristics - Reaction Wheels } \\
\hline inertia $\left(\mathrm{kg} \cdot \mathrm{m}^{2}\right)$ & 0.01911 \\
\hline maximum torque $(N . m)$ & 0.075 \\
\hline maximum angular velocity $(R P M)$ & 6000 \\
\hline \multicolumn{2}{|l|}{ References for the controller } \\
\hline solar vector body (XYZ) & $0]^{T}$ \\
\hline angular velocity $(r / s, \mathrm{XYZ})$ & {$\left[\begin{array}{ll}0 & 0\end{array}\right.$} \\
\hline
\end{tabular}
selected using independent normal distributions $(\mu=$
Table 1: Satellite characteristics and references.

$0, \sigma=1)$ multiplied by $90^{\circ}$; and (2) the initial angular velocity errors are randomly selected using independent normal distributions $(\mu=0, \sigma=1)$ multiplied by $0.01 \mathrm{rad} / \mathrm{s}$.

The Monte Carlo model ran 20 times and in each time one simulation of the two systems was executed. Such executions used simulation time 700 seconds, fixed step 0.01 seconds and the data presented in Table 1.

Fig. 1(a) shows the controllability of the statespace model defined by Equation 13 (using quaternions and the Gibbs vector), whereas Fig. 1(b) shows the controllability of the state-space model defined by Equation 14(MRPs).

Based on Fig. 1, it is possible to conclude that MPRs maximizes the controllability through the simulations. Once MRPs provides better controllability the next step is to further parametrize such statemodel using different SDCs, which is explored in the subsection below. 


\subsection{Parametrization of MRPs}

For multivariable state-space models, as the one studied in the present paper, given two distinct SDC matrices $A_{1}(x)$ and $A_{2}(x)$ then there is an infinite number of SDC parametrizations. Such infinite parametrizations can be constructed using Equation 15 [3].

$$
A(x, \alpha)=\alpha A_{1}(x)+(1-\alpha) A_{2}(x)
$$

Focusing on MRPs, which provides better controllability as evaluated earlier, and taking into account the rule of thumb that the selection of the state-dependent factorization should place a nonzero entry in the $\{i, j\}$-element of the $A(x, \alpha)$ matrix if the $i^{t h}$ state derivative depends on the $j^{\text {th }}$ state [3], the state-space model in Equation 16 is defined (it is based on algebraic manipulations of Equation 7 ).

$$
\begin{array}{r}
{\left[\begin{array}{c}
\dot{x_{3}} \\
\dot{x_{2}}
\end{array}\right]=\left[\begin{array}{cc}
0 & \frac{1}{4}\left(1-p^{T} p\right) I_{3 \times 3}+\frac{1}{2} p^{\times}+\frac{1}{2}\left(p p^{T}\right) I_{3 \times 3} \\
0 & -I_{b}^{-1} \omega^{\times} I_{b}+I_{b}^{-1}\left(\sum_{n=1}^{3} h_{w, n} a_{n}\right)^{\times}
\end{array}\right]\left[\begin{array}{c}
x_{3} \\
x_{2}
\end{array}\right]+\left[\begin{array}{c}
0 \\
-I_{b}^{-1}
\end{array}\right]\left[u_{1}\right]} \\
{[y]=I\left[\begin{array}{l}
x_{3} \\
x_{2}
\end{array}\right]}
\end{array}
$$

Fig. 2 shows the controllability of the state-space model defined by Equation 16 based on MRPs applying a SDRE controller $(Q=1$ and $R=1)$. Controllability is better than the one shown in Fig. 1(a) so MRPs has still better controllability than quaternions with Gibbs vector (Equation 13). Moreover, this second option based on MRPs (Equation 16) has bet- ter controllability than the first one defined by Equation 14

Equation 14 and Equation 16 provide two distinct parametrizations for the same system, therefore, it is possible to use Equation 15 to evaluate if the combination of these two equations provides even better controllability. Equation 17 shown the parametrizations to be used in $A$.

$$
\begin{gathered}
A_{1_{x_{3}}}\left(x_{3}\right)=\left[\begin{array}{ll}
-\frac{1}{2} \omega^{\times}+\frac{1}{2}\left(\omega^{T} p\right) I_{3 \times 3} & \frac{1}{4}\left(1-p^{T} p\right) I_{3 \times 3}
\end{array}\right] \\
A_{2_{x_{3}}}\left(x_{3}\right)=\left[\begin{array}{ll}
0 & \frac{1}{4}\left(1-p^{T} p\right) I_{3 \times 3}+\frac{1}{2} p^{\times}+\frac{1}{2}\left(p p^{T}\right) I_{3 \times 3}
\end{array}\right]
\end{gathered}
$$

Applying parametrizations defined in Equation 17 into Equation 15, a simulation test was conducted with a full Monte Carlo perturbation model, in which $\alpha$ was randomly selected in the interval $0 \leq \alpha \leq 1$. The goal was to evaluate the impact of $\alpha$ based on a given satellite characteristics, initial conditions and references in Table 1

The Monte Carlo model ran 90 times and in each time one simulation using a different $\alpha$ was performed for the same data in Table 1 Fig. 3 shown the resulting controllability of each run. It is possible to conclude that parametrization defined by Equation 16 is the optimal since the controllability is the highest through the entire simulation when $\alpha=0$ (highlighted in the legend, when only $A_{2}$ in Equation 17 is active).

Therefore, the state-space model defined by Equation 16, which results for the controllability is shown in Fig. 2, is the optimal factorization for the SDRE technique applied to the AOCS as described in the current work. Such conclusion is based on the char- acteristics of the satellite, the initial conditions, the references for the controller in Table 1 and the Monte Carlo perturbation model so it neither valid for the general case nor even for a different initial condition out of the range of the Monte Carlo perturbation model. Moreover, as Equation 16 is defined using MRPs, which has singularity for $360^{\circ}$, it is neither unique nor global, whereas the Equation 13 based on quaternions with Gibbs vector is global but not unique.

\section{Simulation Comparison}

Numerical simulations were performed to confirm that the state-space model described by Equation 16 is optimal for the given satellite characteristics, initial conditions and references in Table11 In order to compare the performance, another full Monte Carlo perturbation model was conducted with three independent systems each one defined by equations 13, 14 and 16 applying a $\operatorname{SDRE}$ controller $(Q=1$ and $R=1)$. The Monte Carlo perturbation model is described as follows: (1) the initial Euler angle errors of the non- 


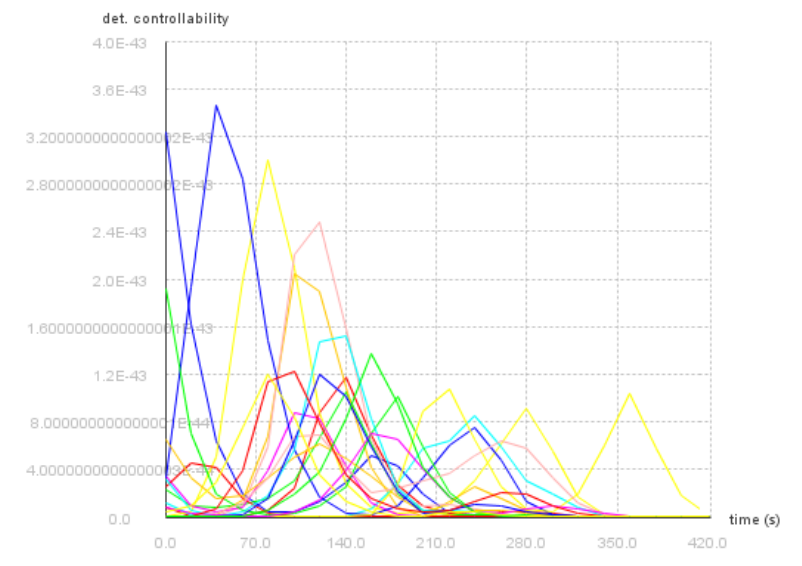

(a) Controllability of state-space model defined using quaternions with Gibbs vector (Equation 13 .

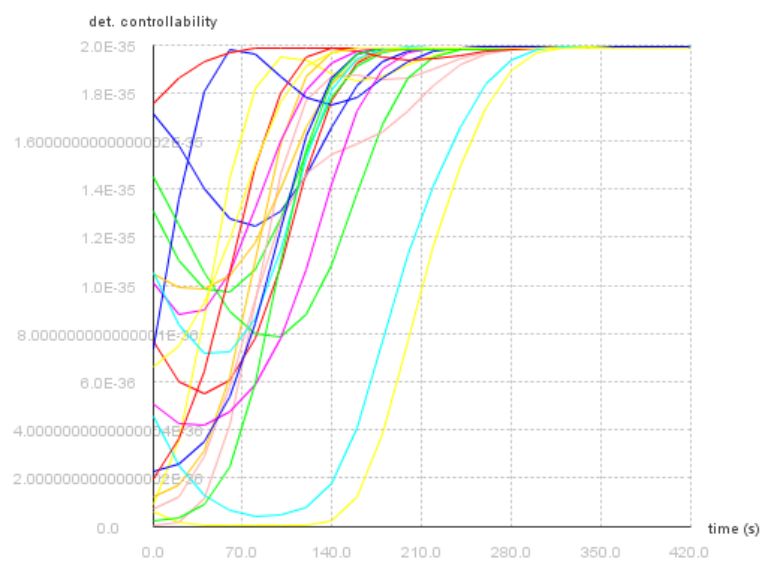

(b) Controllability of state-space model defined using MRPs (Equation 14.

Figure 1: Comparison between state-space models.

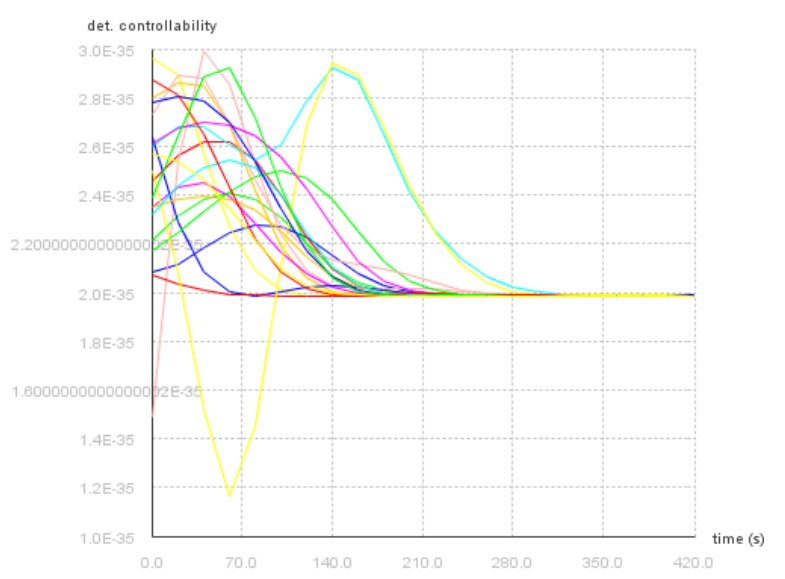

Figure 2: Controllability of state-space model defined using MRPs (Equation 16).

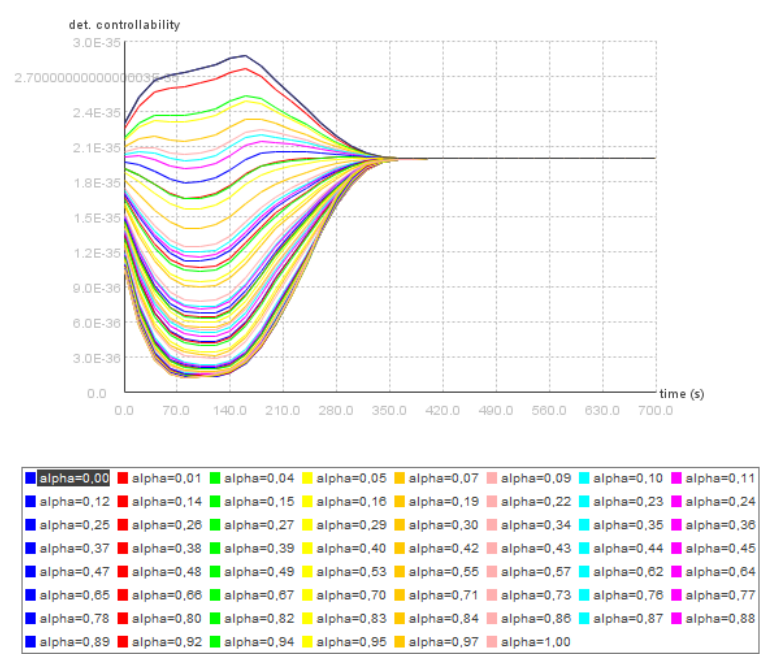

Figure 3: Controllability of state-space model defined using MRPs (Equations 14 and 16 parametrized).

linear spacecraft system are randomly selected using independent normal distributions $(\mu=0, \sigma=1)$ multiplied by $90^{\circ}$; and (2) the initial angular velocity errors are randomly selected using independent normal distributions ( $\mu=0, \sigma=1)$ multiplied by $0.01 \mathrm{rad} / \mathrm{s}$.

The Monte Carlo model ran 20 times and in each time one simulation of the three systems was executed. Such executions used simulation time 700 seconds, fixed step 0.01 seconds and the data presented in Table 1

Fig. 4 shows that the scalar part of quaternion $\left(q_{4}\right)$ converges to $1\left(q_{4} \rightarrow 1\right)$ as well as that the vectorial part of the quaternion converges to $0\left(\left[q_{1} q_{2} q_{3}\right]^{T} \rightarrow\right.$ $\left[\begin{array}{lll}0 & 0 & 0\end{array}\right]^{T}$ ) in the sense that attitude of the satellite reached the target attitude. It is clear that the model applying quaternion with Gibbs (Fig. 4(a) exhibits the worst performance, nonetheless, the two models applying MRPs have similar results (Fig. 4(b) and Fig. 4(c)).

Focusing on the effort to control the system and taking into account that reaction wheels angular velocity must not converge to a determined velocity, the norm of the set of reaction wheels angular momentum is shared in Fig. 5. Since angular momentum $\left(\mathrm{Kgm}^{2} / \mathrm{s}\right)$ is a conservative quantity the changes in such norm indicate how much torque was applied.

Once again it is clear that the model applying quaternion with Gibbs (Fig. 5(a)) exhibits the worst performance since it has the highest peak in the norm (over $18 \mathrm{Kgm}^{2} / \mathrm{s}$ ). Although the two models applying MRPs have similar results (Fig. 5(b) and Fig. 5(c)), it is possible to note that second peak is 


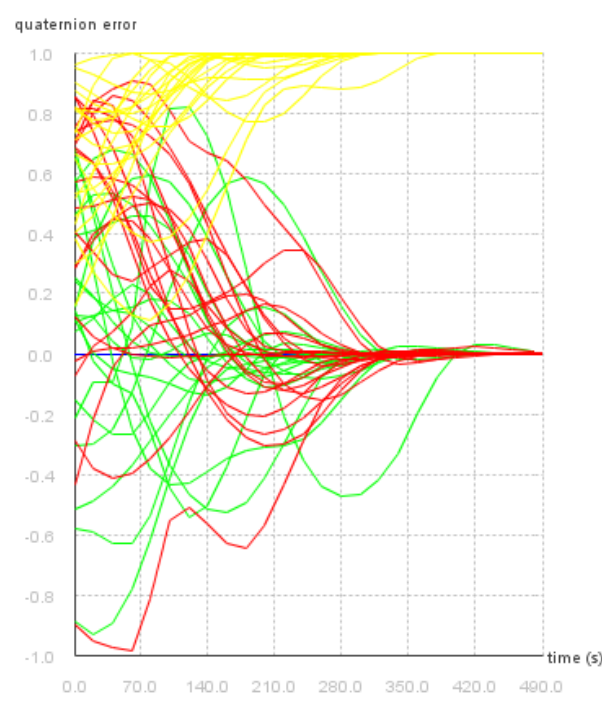

(a) Quaternion error using quaternions with Gibbs vector (Equation 13).

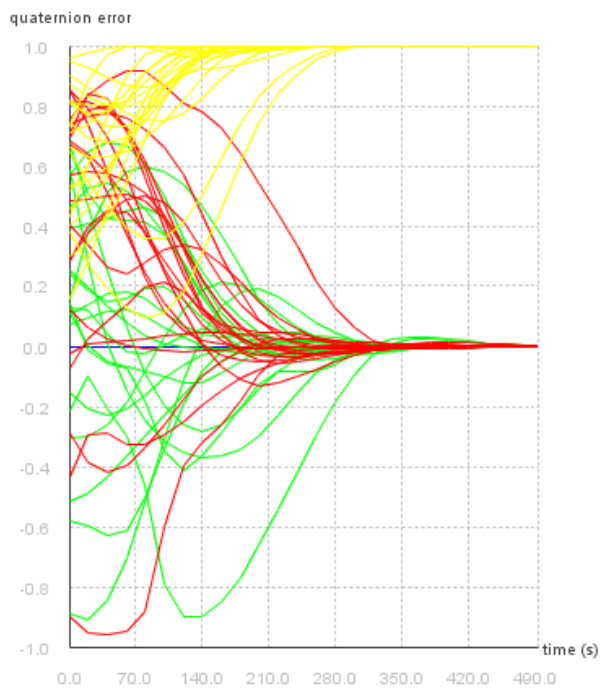

(b) Quaternion error using MRPs (Equation 14).

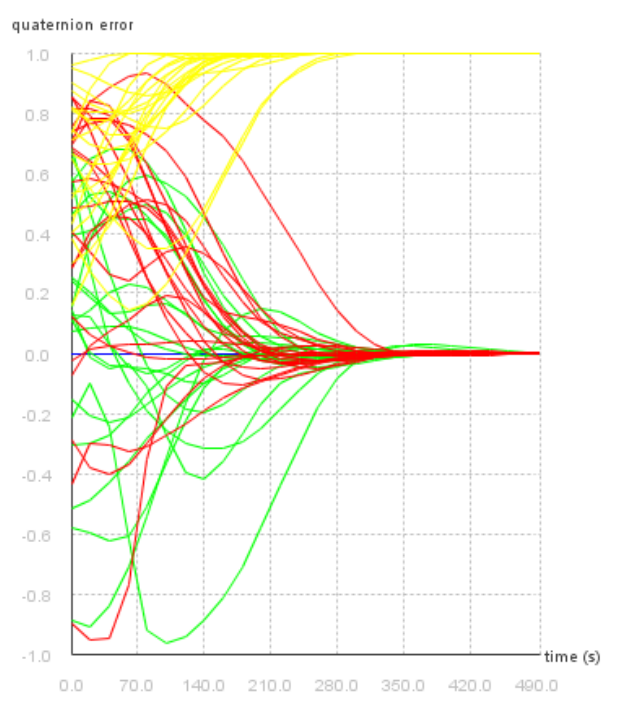

(c) Quaternion error using MRPs (Equation 16.

Figure 4: Comparison between quaternion errors.

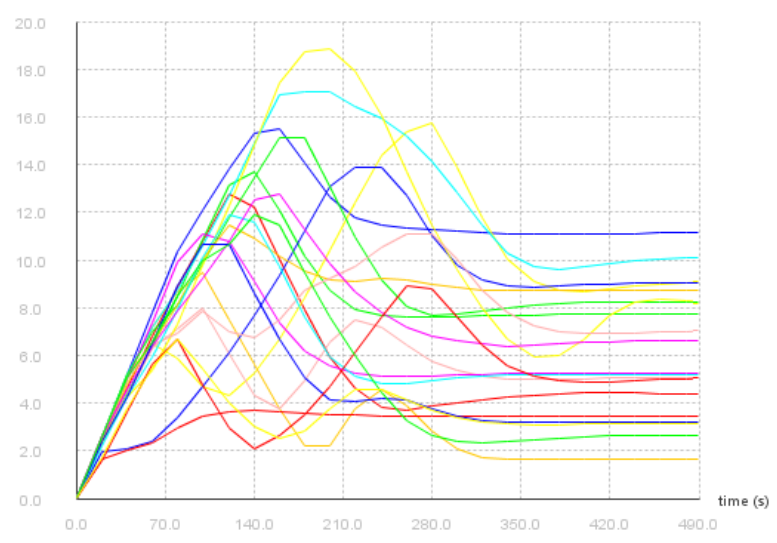

(a) Norm of reaction wheels angular momentum using quaternions with Gibbs vector (Equation 13 .

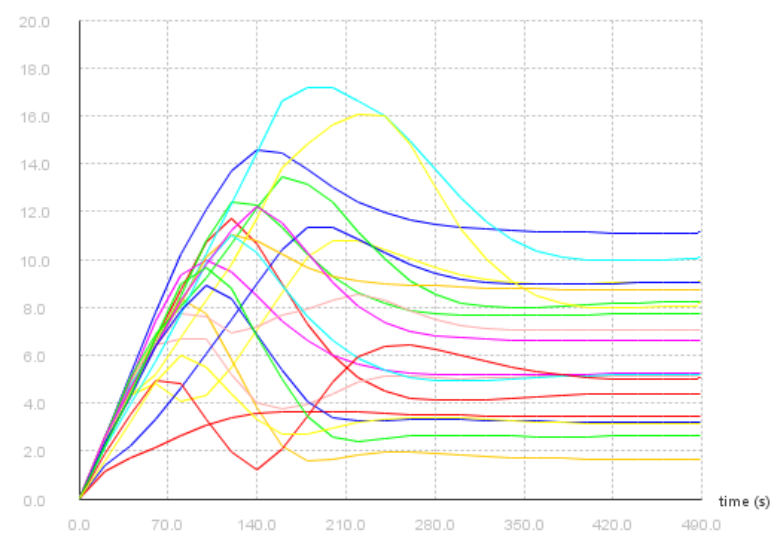

(b) Norm of reaction wheels angular momentum using MRPs (Equation 14].

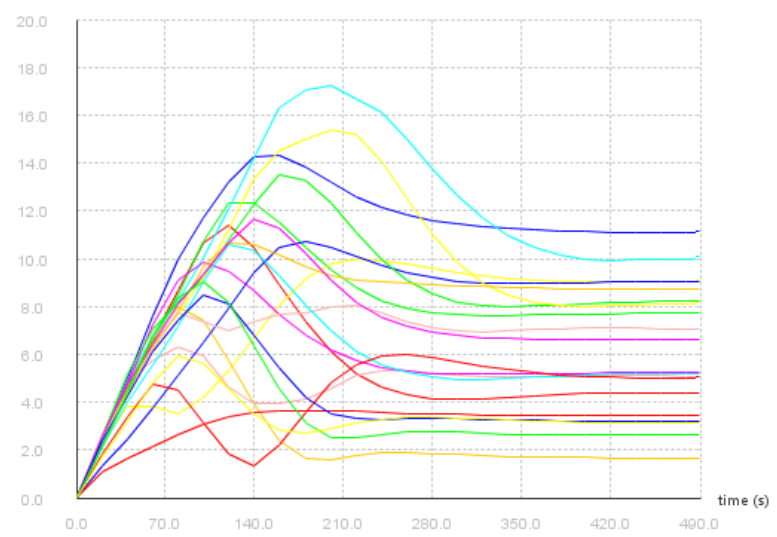

(c) Norm of reaction wheels angular momentum using MRPs (Equation 16 .

Figure 5: Comparison between norms of reaction wheels angular momentum. 
lower in the Fig. 5(c), indicating that it is saving torque, and, consequently, energy.

Such simulations results are neither valid for the general case nor for initial conditions out of the range of the Monte Carlo perturbation models due to the underlining nonlinear dynamics.

\section{Conclusion}

To the best of our knowledge, this is an original contribution for the optimal arrangement of the SDC for a three-axis stabilized satellite model. The results shown that different SDCs can produce extremely different results ranging from non-applicability of the SDRE technique to differences in the controllability and, consequently, in the performance and robustness of the system. Unfortunately, the optimal factorization found is neither valid for the general case nor even for different initial conditions out of the range of the Monte Carlo perturbation model due to the underlining nonlinear dynamics. However, the procedure applied can provide guidance for engineers.

A secondary contribution is the kinematical part of the state-space models in equations 13, 14 and 16 since they can be used in any system that exhibit rotational motion, e.g., airplanes. The simulations were performed using a portable simulator developed at INPE [9, 12], such simulator has capabilities for the unloading of the angular momentum of the reaction wheels (based on a magnetometer and a set of magnetorques) not explored in the current paper due to the lack of space. Furthermore, this simulator is heavily based on Hipparchus [13].

Regarding the discussion whether the SDC factorization of SDRE technique in AOCS can yield gains in the missions developed by INPE, since performance in the LEOP is critical to the success of a mission and the simulation results show that the performance and robustness of SDRE controllers can be enhanced by optimal factorizations (in particular, with kinematics based on MRPs); SDRE can yield gains. Nonetheless, its implementation requires more computing resources and tend to exhibit difficulties for verification. Therefore, it is too early to draw a definitive conclusion.

In conclusion, the optimal factorization of SDC in the SDRE technique is of utmost importance for performance and robustness of nonlinear systems controlled by such technique.

\section{References:}

[1] J. D. Pearson. Approximation Methods in Optimal Control I. Sub-optimal Control†. Journal of Electronics and Control, 13(5):453--469, 1962.
[2] Tayfun Çimen. State-Dependent Riccati Equation (SDRE) control: A survey. IFAC Proceedings Volumes (IFAC-PapersOnline), 17(1 PART 1):3761--3775, 2008.

[3] Tayfun Çimen. Systematic and effective design of nonlinear feedback controllers via the statedependent Riccati equation (SDRE) method. Annual Reviews in Control, 34(1):32--51, 2010.

[4] James R Cloutier, Christopher N D'Souza, and Curtis P Mracek. Nonlinear regulation and nonlinear H-infinity control via the state-dependent Riccati equation technique. Conference on Nonlinear Problems in Aviation, (September), 1996.

[5] R G Gonzales and Luiz Carlos Gadelha de Souza. Application of the sdre method to design a attitude control system simulator. $A d$ vances in the Astronautical Sciences, 134(Part 1-3):2251--2258, 2009. Setores de Atividade: Educação.

[6] G Di Mauro, M Schlotterer, S Theil, and M Lavagna. Nonlinear Control for Proximity Operations Based on Differential Algebra. Journal of Guidance, Control, and Dynamics, 38(11):2173--2187, apr 2015.

[7] Giuseppe Di Mauro, Pierluigi Di Lizia, and Michelle Lavagna. Control of Relative Motion via State-Dependent Riccati Equation Technique. In AAS/IAAA Astrodynamics Specialist Conference, Vol 142, Univelt, San Diego, Ca, 2011.

[8] P. K. Menon, T. Lam, L. S. Crawford, and V. H.L. Cheng. Real-time computational methods for SDRE nonlinear control of missiles. Proceedings of the American Control Conference, 1:232--237, 2002.

[9] Alessandro Gerling Romero and Luiz Carlos Gadelha de Souza. State-dependent riccati equation controller using java in remote sensing cubesats. Journal of Applied Remote Sensing, 13(3):e032509, July 2019.

[10] Peter C Hughes. Spacecraft Attitude Dynamics. New York, 1986.

[11] Marcel J Sidi. Spacecraft Dynamics and Control - A Practical Engineering Approach. Cambridge University press, 2006.

[12] Alessandro Gerlinger Romero. Satellite simulation developer's guide - attitude dynamics and control of nonlinear satellite simulations. Instituto Nacional de Pesquisas Espaciais, São José dos Campos, 2020. 
[13] Hipparchus (version 1.3-snapshot), 2018. Access date: 28.Jan.2018.

\section{Contribution of individual authors to} the creation of a scientific article

\section{(ghostwriting policy)}

Alessandro Gerlinger Romero carried out the modeling, the code developing, the simulation and the writing.

Luiz Carlos Gadelha de Souza oriented the first author and contributed in the writing.

\section{Creative Commons Attribution}

\section{License 4.0 (Attribution 4.0}

\section{International, CC BY 4.0)}

This article is published under the terms of the Creative Commons Attribution License 4.0

https://creativecommons.org/licenses/by/4.0/deed.en_US 